\title{
Non-linear Modelling of Fasting Plasma Glucose Concentration and Peak 2-hour Insulin Response to Glucose Challenge in Australian Aboriginal People
}

\author{
Mark Daniel ${ }^{1,2,3^{*}}$, Geng Zang ${ }^{3}$, Kevin G. Rowley ${ }^{2,4}$, Robyn McDermott ${ }^{1}$, Shona J. Kelly ${ }^{1}$ and Kerin O'Dea ${ }^{1,2}$ \\ Correspondence: mark.daniel@unisa.edu.au \\ 'Sansom Institute for Health Research, University of South Australia, Adelaide, Australia. \\ 2Department of Medicine, St. Vincent's Hospital, The University of Melbourne, Australia. \\ ${ }^{3}$ Centre hospitalier de I'Université de Montréal, Université de Montréal, Canada. \\ ${ }^{4}$ Onemda VicHealth Koori Health Unit, Centre for Health and Society, Melbourne School of Population Health, \\ The University of Melbourne, Australia.
}

\begin{abstract}
Background: The aim of this study was to investigate the relationship between 2-hour insulin concentration and fasting plasma glucose concentration (FPG) in a population-based sample of Indigenous Australians.

Methods: Data collected from 2930 adults with unknown diabetic status were analyzed using three non-linear modeling methods: locally weighted regression (LOESS), generalized additive models (GAM), and fractional polynomial (fracpoly) regression procedures.

Results: Log fasting insulin and log 2-hour insulin had nonlinear relationships with FPG. All models indicated a consistent fit for 2-hour insulin response across FPG values of 3.5-5.8 mmol/I. GAM and fracpoly regressions overlapped across FPG values of 3.5-13 mmol/l. The LOESS model had a slightly different pattern from FPG of 5.8-17 mmol/l. For all models, log 2-hour insulin concentration increased across FPG values from $3.5-7.0 \mathrm{mmol} / \mathrm{l}$ and decreased for FPG $>7.0 \mathrm{mmol} / \mathrm{l}$.

Conclusions: The $7.0 \mathrm{mmol} / \mathrm{I} \mathrm{FPG} \mathrm{diagnostic} \mathrm{cut-off} \mathrm{represents} \mathrm{the} \mathrm{start} \mathrm{of} \mathrm{a} \mathrm{diminishing} \mathrm{second-phase} \mathrm{insulin} \mathrm{response} \mathrm{to}$ glucose, indicating that pancreatic output begins to decline at this FPG level. These results provide strong physiological rationale, beyond rising rates of clinical complications, for the revised fasting glucose diagnostic criterion of $7.0 \mathrm{mmol} / \mathrm{l}$. Further research is needed to evaluate whether similar relationships exist for other high- and low-risk populations.

keywords: epidemiology; biostatistics; screening, Australian Aboriginal people, insulin
\end{abstract}

\section{Introduction}

The current American Diabetes Association recommended diagnostic criterion for type 2 diabetes mellitus of fasting plasma glucose $(F P G) \geq 7.0 \mathrm{mmol} / \mathrm{l}$ is based on the point at which risk for macrovascular and microvascular complications substantially increases [1,2]. The WHO continues to recommend assessment of plasma glucose level by oral glucose tolerance test (OGTT) with a $75 \mathrm{~g}$ glucose load and the diagnostic criterion of a 2-hour post-challenge plasma glucose $\geq 11.1 \mathrm{mmol} / \mathrm{I}$ [2]. The OGTT with glucose loading and two venipunctures is unpopular with patients and screening test participants, and is difficult to implement in field settings. This is so for Australian Aboriginal peoples who, with diabetes prevalence rates of $15-30 \%[3,4]$ and incidence rates of 20-23 cases per 1,000 person-years $[5,6]$, are at elevated risk for having and developing the disease.

Specific aetiologies of type 2 diabetes mellitus and the exact contributions that insulin secretion and insulin sensitivity play in hyperglycaemia associated with type 2 diabetes, are not fully understood [1]. The relationship is hyperbolic: at early stages of type 2 diabetes, insulin secretion increases as the body responds to elevated blood glucose levels and then, as blood glucose levels continue to be elevated or increase further, insulin secretion decreases (i.e., "Starling's curve of the pancreas") [7-9]. This evidence has shaped the theory that the primary abnormality in type 2 diabetes is insulin resistance and $\beta$-cell dysfunction is a secondary impairment [7].

Insulin secretory response to oral glucose challenge has been shown to correlate with 2-hour plasma glucose levels. Previous studies have indicated that insulin responses rise and peak between 2-hour plasma glucose levels of 7.8-11.1 $\mathrm{mmol} / \mathrm{l}$ and become progressively lower for 2-hour plasma glucose levels greater than $11.1 \mathrm{mmol} / \mathrm{l}[10,11]$. Such data have provided physiological support for the WHO-recommended type 2 diabetes diagnostic criterion of plasma glucose $\geq 11.1$

(c) 2012 Daniel et al; licensee Herbert Publications Ltd. This is an open access article distributed under the terms of Creative Commons Attribution License (http://creativecommons.org/licenses/by/3.0), This permits unrestricted use, distribution, and reproduction in any medium, provided the original work is properly cited 
$\mathrm{mmol} / \mathrm{l}$ after OGTT.

The underlying physiological mechanisms by which the above relationships are expressed for different levels of fasting glucose have not been resolved. Studies of the association between insulin secretion and FPG have produced varied and contradictory results. Previous studies have described the distribution of fasting insulin as a function of FPG, finding a peak of fasting insulin at FPG $7.8 \mathrm{mmol} / \mathrm{l}[\mathbf{7 , 9 , 1 2 ]}$. For first-phase insulin secretion, other work has found a marked decline between FPG of 5.0 to $5.4 \mathrm{mmol} / \mathrm{I}$ [13] while still other studies suggest no clear threshold at which early insulin secretion is reduced [14]. Insulin secretion in response to 2-hour OGTT has been shown to decrease in FPG greater than $6.1 \mathrm{mmol} / \mathrm{I}$ [7] or, in a comparison of ethnic groups, between 7.1 and 7.8 $\mathrm{mmol} / \mathrm{l}[15]$.

Little population-based research has addressed insulin response in relation to FPG levels to better understand the connections between a threshold FPG level and physiological changes in insulin secretion and sensitivity. An improved understanding of the changes in insulin that correspond to a spectrum of fasting glucose, and hyperglycaemia in particular, could provide an aetiological basis to complement FPG thresholds which correlate with adverse outcomes. In this study, we evaluated the relationship between FPG and fasting insulin and 2-hour post-challenge insulin concentrations based on OGTTs in Aboriginal Australians at various stages in the progression of the disease but not known to have existing diabetes.

\section{Patients and Methods \\ Population and setting}

Data were collected in community-based screening for diabetes and coronary risk factors in 25 Aboriginal Australian settlements between 1983 and 1997. Screening test analyses [16] and results have been reported previously [3,5,17-21]. Participation was voluntary for all persons at least 15 years of age with no known disease. Response rates ranged from $54-90 \%$ of resident adults present in the community during screening; participants ranged from 57 to 582 across settlements (median=82). The distribution of age by sex was representative of that for the national Australian Aboriginal population $[3,22]$. Review board approval was provided by the institutions responsible during data collection: Alice Springs Institutional Ethics Committee and ethics committees at the University of Melbourne and Deakin University, Melbourne, Australia. All participants provided their informed, written consent.

Study communities were located in central, north-western and north-eastern Australia, representing the spectrum of 'remote' settings in which Aboriginal Australians live, from central desert areas to tropical, monsoonal regions in the north. Lifestyles in these communities are mixes of traditional and, increasingly, western ways of living (i.e., poor diet and inactivity) related to development of diabetes and cardiovascular disease.

\section{Study Measures}

FPG and fasting insulin were measured from a venous blood sample collected following an overnight fast. OGTTs were given with participants ingesting a 75-gram carbohydrate load. A second blood sample was drawn 2 hours later. Blood samples were transferred to fluoride-heparin tubes and kept cold until centrifugation and separation of plasma at $2500 \mathrm{rpm}$. Plasma was stored prior to automated analysis of glucose by glucose oxidase or hexokinase methods (Boehringer-Mannheim, Sydney). Insulin was determined by radio-immunoassay (pre-1993 data: Pharmacia Diagnostics; Uppsala, Sweden, inter-assay CV 5\%; 1993 and later data: Linco Research, Inc., St. Louis, U.S., inter-assay CV $<14 \%$ at lower levels, $\leq 9 \%$ in the mid-range, and $\leq 5 \%$ in the upper working range). An early analysis of 16 persons included in the present sample showed median differences of $6.8 \%$ between assays $(r=0.98)$ [23] but, as noted in the Results, the distribution of insulin did not differ between the two assays.

\section{Data Analysis}

The relationships between FPG and fasting insulin and 2-hour post-challenge insulin were modelled with participants pooled, thus including a spectrum of status from normoglycaemia to diabetes (but not including individuals already known to have diabetes). As the shape of the relationship between insulin and glucose was of interest, specifically, the inflection point, non-linear modelling methods were used. The use of multiple methods of analysis allowed us to assess consistency between different methods and identify model specification bias. Different modelling methods were thus used to assess whether a given inflection point was specific to a particular statistical approach, or if results converged for different modelling strategies. Natural logarithm transformations of fasting and 2-hour insulin were used to reduce non-normality and heteroscedasticity of errors.

A first set of analyses was conducted with locally weighted polynomial regression (LOESS) which fits simple models to localised subsets of data. These are combined into a function that describes the relationship between the variables. Analysis was conducted using SAS PROC LOESS (SAS 9.1.3). The regression model used local quadratic fitting, local least squares fitting for every point in the dataset, and three reweighted iterations. Automatic smoothing parameters were used: 0.4 for the fasting insulin regression model; and 0.8 for the 2-hour insulin regression model.

A second set of analyses used a nonparametric generalised additive model (GAM) procedure. This model assumes that the mean of the outcome variable depends on an additive predictor estimated through a nonlinear link function. An iterative estimation process completes when the likelihood of the data, given the model, cannot be improved. We conducted this analysis using SAS PROC GAM (SAS 9.1.3). Six models were fitted with smoothing splines of $4,5,6,7,8$ and $9 d f$ specified 
for fasting insulin, and 2, 3, 4, 5, 6 and $7 d f$ for 2-hour insulin.

A third set of analyses used fractional polynomial regression. This procedure fits a series of models where the relationship is presumed to be first order fractional polynomial. Different fractional polynomial models are estimated; that with the best fit is identified using criteria to measure model goodness of fit. We used Stata fracpoly regress (Stata 8.2) to fit six fractional polynomial regression models for $4,5,6,7,8$ and 9 $d f$ for fasting insulin, and 2, 3, 4, 5, 6 and $7 d f$ for 2-hour insulin.

The Akaike Information Criterion was used to evaluate the best fit for GAM and fracpoly analyses using the equation Akaike Information Criterion=Deviance $+2^{*} p$, where $p$ is the number of parameters $(d f)$ in the model. A lower criterion value indicates a better fit.

\section{Results}

A total of 2958 observations were available to analyse the relationship between insulin and FPG, from which 28 were excluded due to 2 -hour insulin concentration values $>3000$ $\mathrm{pmol} / \mathrm{l}$ and/or FPG values $<3.0 \mathrm{mmol} / \mathrm{l}$. The $25^{\text {th }}, 50^{\text {th }}$ and $75^{\text {th }}$ percentile values for 2-hour insulin (pmol/l) were 156, 288 and 516 for pre-1993 data (Pharmacia assay) $(n=1602)$ and 138, 270 and 516 for 1993 and later data (Linco assay) (n=1328). As such, the distributions of insulin did not differ between assays, and there was no difference in means ( $t_{2928 \mathrm{df}}=0.04, p=0.97$ ). Table 1 gives descriptive statistics and biochemical data for the 2930 individuals included in analyses. Ten percent of the sample was classified as having impaired glucose tolerance and $11.8 \%$ were classified as having diabetes.

In relation to log fasting insulin, the first quartile of FPG $(\mathrm{mmol} / \mathrm{l})$ was 4.3 , the median was 4.8 and the third quartile was 5.4. Ninety percent of FPG observations were below 6.9. In relation to log 2-hour insulin, the first quartile of FPG $(\mathrm{mmol} / \mathrm{l})$ was 4.3 , the median was 4.7 , and the third quartile was 5.3. Eighty percent of FPG observations were between 3.5 and 5.8 , and $90 \%$ were below 7.0. Figure 1 provides the scatter plot for log 2-hour insulin and FPG observations, illustrating non-linearity and considerable variability.

Akaike Information Criterion values, indicating the best fit for GAM and fracpoly models, are presented in Table 2 according to degrees of freedom for analyses of log fasting and $\log 2$-hour insulin versus FPG values. For log fasting insulin, the GAM analysis with $d f=6$ had the best fit amongst the six models tested, each with a different smoothing spline for different degrees of freedom, and the fracpoly analysis with $d f=9$ had the best fit. For 2-hour insulin, the GAM analysis with $d f=5$ and the fracpoly analysis with $d f=4$, were the best fits.

For the three regression models using LOESS, GAM $(d f=6)$, and fracpoly $(d f=9)$ methods, peak log fasting insulin corresponded to FPG of $7.1 \mathrm{mmol} / \mathrm{l}$ for the LOESS model, 7.5 $\mathrm{mmol} / \mathrm{l}$ for the GAM model, and $7.3 \mathrm{mmol} / \mathrm{l}$ for the fracpoly model (data not shown). The three methods produced a nearly consistent fit, with the exception of the fracpoly model which fluctuated along the GAM and LOESS lines at FPG $>8.0 \mathrm{mmol} / \mathrm{l}$.
Table 1. Descriptive characteristics and biochemical data for 2930 Aboriginal Australians.

\begin{tabular}{lcccc}
\hline \multirow{2}{*}{ Variable } & Mean & $\begin{array}{c}\text { Std } \\
\text { Dev }\end{array}$ & $\begin{array}{c}\text { Mini- } \\
\text { mum }\end{array}$ & $\begin{array}{c}\text { Maxi- } \\
\text { mum }\end{array}$ \\
\cline { 2 - 3 } Age (years) & 35.2 & 15.9 & 15.0 & 84.0 \\
Sex Men (\%) & 46.3 & 0.92 & - & - \\
$\quad$ Women (\%) & 53.7 & 0.92 & - & - \\
Body mass index (kg/m²) & 26.1 & 6.2 & 15.3 & 55.1 \\
Fasting plasma glucose (mmol/l) & 5.24 & 2.03 & 3.00 & 19.60 \\
Two-hour glucose (mmol/l) & 7.01 & 4.13 & 2.90 & 32.00 \\
Fasting insulin (pmol/l) & 110.7 & 97.0 & 12.0 & 1500.0 \\
Two-hour insulin (pmol/l) & 393.9 & 377.2 & 12.0 & 2970.0 \\
Normoglycemic (\%) & 78.2 & 0.76 & - & - \\
Impaired glucose tolerance (\%) & 10.0 & 0.55 & - & - \\
Diabetes (\%) & 11.8 & 0.60 & - & - \\
\hline
\end{tabular}

Table 2. Akaike Information Criterion values according to degrees of freedom for non-parametric fitted models of log fasting and log 2-hour insulin versus fasting plasma glucose.

\begin{tabular}{lcccccc}
\hline \multicolumn{7}{c}{ Degrees of freedom } \\
\hline $\begin{array}{l}\text { Log fasting } \\
\text { insulin }\end{array}$ & 4 & 5 & 6 & 7 & 8 & 9 \\
GAM & 1224.5 & 1222.8 & 1222.5 & 1223.0 & 1224.0 & 1225.3 \\
fracpoly & 6061.3 & 6059.3 & 6057.8 & 6057.1 & 6052.6 & 6052.4 \\
Log 2-hour & & & & & & \\
insulin & 2 & 3 & 4 & 5 & 6 & 7 \\
GAM & 2122.8 & 2108.3 & 2104.9 & 2104.1 & 2104.5 & 2105.4 \\
fracpoly & 7362.4 & 7336.2 & 7334.8 & 7335.7 & 7337.5 & 7339.2 \\
\hline
\end{tabular}

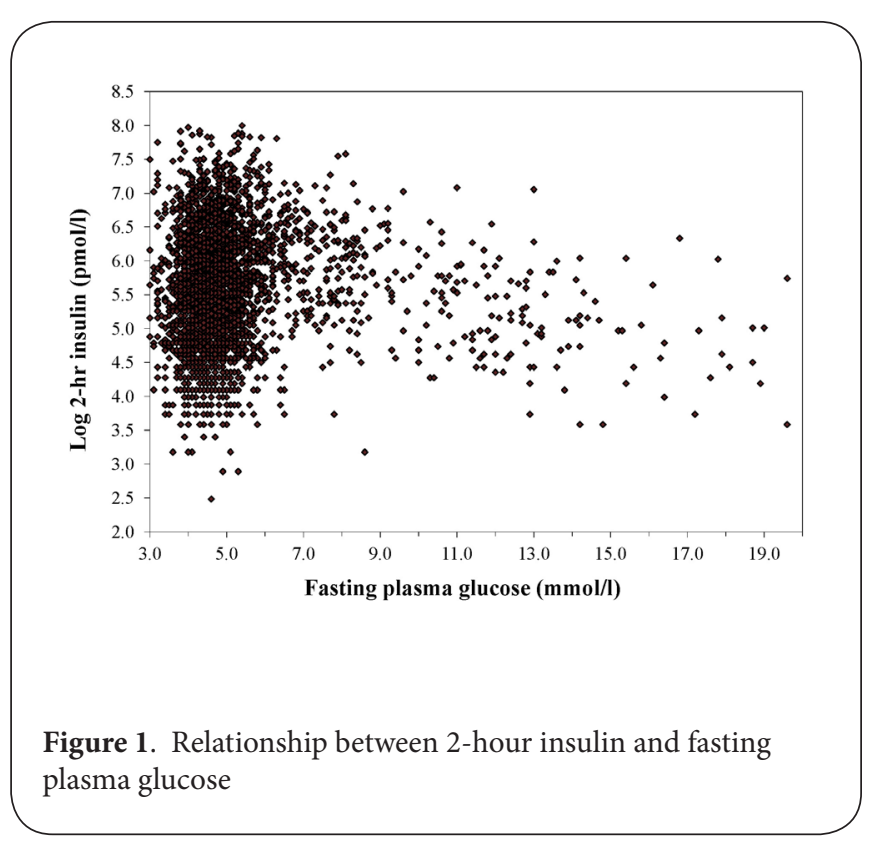


Table 3. Log 2-hour insulin response to oral glucose tolerance test estimated according to fasting plasma glucose values using three nonlinear statistical modelling procedures, with $95 \%$ confidence limits for the fractional polynomial regression procedure

\begin{tabular}{|c|c|c|c|c|c|c|c|}
\hline & \multicolumn{7}{|c|}{ Fasting plasma glucose (mmol/l) } \\
\hline & 6.7 & 6.8 & 6.9 & 7.0 & 7.1 & 7.2 & 7.3 \\
\hline & \multicolumn{7}{|c|}{ Estimated log 2-hour insulin response (pmol/l) } \\
\hline Locally weighted regression (LOESS) & 6.0143 & 6.0177 & 6.0196 & 6.0202 & 6.0198 & 6.0185 & 6.0162 \\
\hline Generalised additive models (GAM) $(d f=5)$ & 5.9421 & 5.9457 & 5.9479 & 5.9487 & 5.9482 & 5.9465 & 5.9436 \\
\hline Fractional polynomial regression (fracpoly) $(\mathrm{df}=4)$ & 5.9457 & 5.9483 & 5.9497 & 5.9499 & 5.9490 & 5.9470 & 5.9440 \\
\hline Lower 95\% confidence limit (fracpoly) & 5.8723 & 5.8735 & 5.8736 & 5.8725 & 5.8704 & 5.8673 & 5.8631 \\
\hline Upper 95\% confidence limit (fracpoly) & 6.0191 & 6.0231 & 6.0258 & 6.0273 & 6.0276 & 6.0267 & 6.0249 \\
\hline
\end{tabular}

Figure 2 illustrates the relationship between log 2-hour insulin and FPG for the regression models using LOESS, GAM $(d f=5)$, and fracpoly $(d f=4)$ methods. Larger differences were apparent between the three plotted lines, for FPG less than 3.5 and greater than $17 \mathrm{mmol} / \mathrm{l}$ where data were sparse. All three methods showed a consistent fit at FPG from 3.5-5.8. The GAM and fracpoly lines overlapped at FPG from 3.5-13. The LOESS line diverged slightly at FPG from 5.8 onward, but ran in close parallel until the extremes of FPG. All three lines show the same pattern: fitted log 2-hour insulin increases from a FPG value of $3.5 \mathrm{mmol} / \mathrm{l}$ until "flattening out" from 6.9-7.1 $\mathrm{mmol} / \mathrm{l}$ at which point a reduction begins.

Estimated log 2-hour insulin values corresponding to FPG from 6.7-7.3 mmol/l, given in Table 3, indicate a peak insulin response at $\mathrm{FPG}=7.0 \mathrm{mmol} / \mathrm{l}$ for all three statistical models. Table 3 gives in addition, the $95 \%$ confidence intervals for estimated log 2-hour insulin for FPG from $6.7-7.3 \mathrm{mmol} / \mathrm{l}$ for the fractional polynomial model (the only method for which confidence intervals could be estimated).

\section{Discussion}

Our analyses demonstrate that for a large population-based sample of Aboriginal Australians, 2-hour post-load insulin concentrations are greatest between fasting plasma glucose values of $6.7-7.3 \mathrm{mmol} / \mathrm{l}$, peaking at $7.0 \mathrm{mmol} / \mathrm{l}$ but with only minor variations in estimated insulin for fasting plasma glucose values of 6.9-7.1 mmol/I (Figure 2, Table 3). The point at which 2-hour insulin begins to decline corresponds to the fasting plasma glucose value at which the risk of macrovascular complications and microvascular risks such as retinopathy are known to increase $[1,2]$. Thus these results provide physiological support for the current diabetes type 2 diagnostic criterion of fasting plasma glucose of $7.0 \mathrm{mmol} / \mathrm{l}$. This conclusion is based on the consistent results of different nonlinear statistical procedures, suggesting it is not an artefact of the modelling process used.

Aboriginal Australians have significantly higher rates of diabetes type 2 than non-Aboriginal Australians [24] and

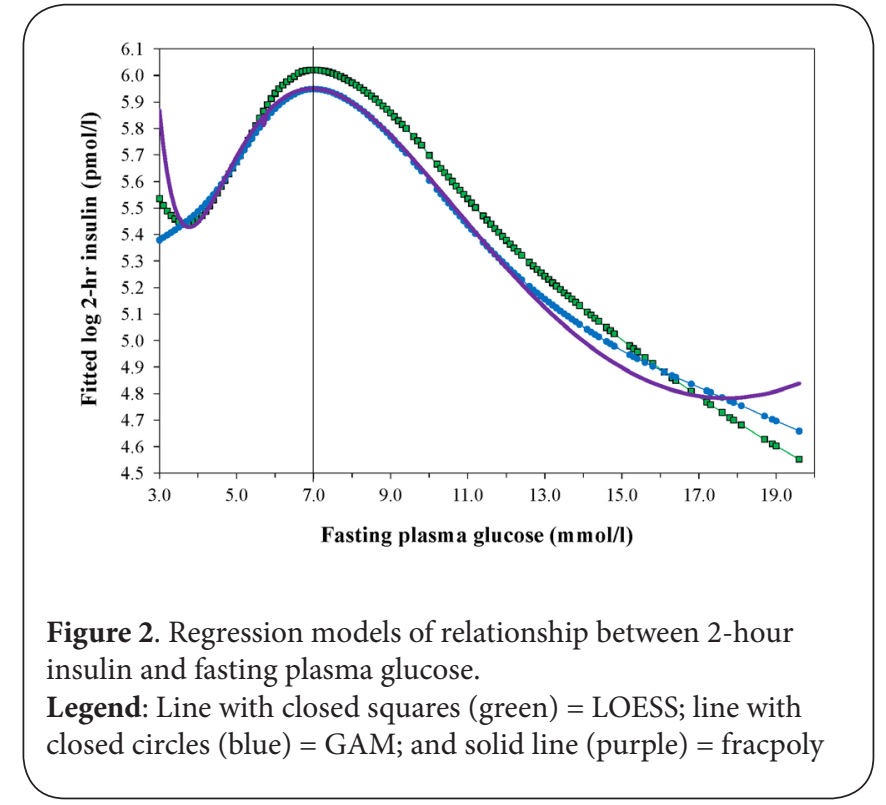

diabetes and related health conditions are recognised as major causes of premature mortality in this population [25]. Early detection and treatment of diabetes type 2 is essential for preventing and reducing the progression of disease complications. As a more efficient and less burdensome procedure than the WHO-recommended OGTT method, the fasting plasma glucose test with a criterion value of $7.0 \mathrm{mmol} / \mathrm{I}$ has advantages for screening in Aboriginal communities.

Other published reports of research that examined the peak of second-phase insulin secretion after an OGTT in relation to fasting plasma glucose values have involved use of less rigorous statistical analysis procedures than applied here, or were based on data from clinical rather than populationbased samples. Dowse et al. (1993), previously reported that 2-hour insulin peaked between 7.1 and $7.8 \mathrm{mmol} / \mathrm{l}$ in population samples of four Mauritian ethnic groups [15], but 
the statistical procedure used was less comprehensive than employed here. Results from clinical research with Japanese patients have shown that 2-hour insulin begins to decline at fasting plasma glucose values of $7.2 \mathrm{mmol} / \mathrm{l}$ [26].

Other research has focused on other aspects of the relationship between insulin and fasting plasma glucose values, primarily examining early insulin secretion during a 2-hour OGTT. Results vary. Gastaldelli et al. (2004), found a peak early insulin response at fasting plasma glucose of 5.6 $\mathrm{mmol} / \mathrm{l}$ in a clinical sample of Mexican-American and Caucasian subjects in Texas [27]. Data from research with Pima Indians indicate that first-phase insulin response declines in individuals with fasting plasma glucose beyond $6.1 \mathrm{mmol} / \mathrm{I}$ [28]. Other analyses of a large clinical sample, however, indicated no definite fasting glucose threshold at which first-phase insulin reductions occurred [14]. Using intravenous administration of glucose, Godsland et al. (2004), found that first-phase insulin secretion begins to decline for fasting plasma glucose between 5.0 and $5.4 \mathrm{mmol} / /[13]$ and that late phase insulin secretion starts to decline at fasting plasma glucose of $6.0 \mathrm{mmol} / \mathrm{l}$ [13].

Clinical research has assessed relationships between fasting plasma glucose and total insulin response to glucose challenge over 2 hours, in contrast to our examination of second-phase insulin response. Total insulin response over the 2 hours following OGTT has been reported by various clinical studies to peak at fasting plasma glucose values from $6.1 \mathrm{mmol} / \mathrm{l}[7,8]$ to $7.2 \mathrm{mmol} / \mathrm{l}$ [26]. Other clinical research using a $100 \mathrm{~g}$ glucose load has demonstrated a peak in total plasma insulin response over 2 hours for fasting plasma glucose of $6.7 \mathrm{mmol} / \mathrm{I}$ [12].

Research examining insulin concentration in relation to the various levels of fasting glucose and categories of glucose tolerance, corresponding to designated ranges of fasting glucose as defined by the American Diabetes Association [29], has yielded a range of results. Several studies have found early insulin response to be notably reduced for impaired fasting glucose (IFG) (fasting glucose $5.6-6.9 \mathrm{mmol} / \mathrm{l}$ and $2-\mathrm{h}$ glucose $<7.8 \mathrm{mmol} / \mathrm{l}$ ) and impaired glucose tolerance (IGT) (fasting glucose $<5.6 \mathrm{mmol} / \mathrm{l}$ and 2-h glucose $7.8-11.1 \mathrm{mmol} / \mathrm{l}$ ) categories, compared to individuals with normal glucose tolerance (NGT) (fasting glucose $<5.6 \mathrm{mmol} / \mathrm{l}$ and 2-h glucose $<7.8 \mathrm{mmol} / \mathrm{l}$ ) [30-34]. Research on 2-hour insulin concentration has shown higher levels in IFG, IGT, and IFG/IGT groups than in NGT and higher concentration in IGT than IFG individuals $[27,34]$. Other work has shown no difference in mean insulin response for 3 hours following OGTT between subjects with NGT and those with diabetes type 2 with FPG $>8.0 \mathrm{mmol} / \mathrm{l}$, but higher insulin responses in individuals with diabetes type 2 with FPG $<8.0 \mathrm{mmol} / \mathrm{l}$ and highest insulin responses in individuals with IGT [35]. For overall insulin secretion, a study with Pima Indians indicated that an increase in total insulin response over 3 hours was much higher for subjects with IGT than for those with either NGT or diabetes type 2 [28]. Other work indicates that overall insulin response is slightly reduced in IFG and for persons with mild diabetes type 2 [36].
This study has a number of limitations, in spite of its large sample of nearly 3000 individuals and the multiple statistical methods employed which differentiate it from clinical studies and less rigorous population analyses. Substantial variation in 2-hour insulin values restricts the certitude with which the relationship shown by our modelling can be applied to individuals. The 2-hour insulin value is an empirical measure of stimulated insulin secretion and could vary depending on, for example, glucose absorption patterns or the effectiveness of incretin factors. Such influences might account for large variations observed in 2-hour insulin. The alignment of all three nonlinear estimation procedures indicating peak insulin response at a fasting plasma glucose of $7.0 \mathrm{mmol} / \mathrm{l}$, and $95 \%$ confidence intervals for the estimated peak of insulin concentration (Table 3), suggest reasonable assurance, however, in our conclusions.

In the context of the community screening initiatives by which our data were gathered, non-compliance with fasting instructions is possible. Our focus on Aboriginal Australians may also limit the applicability of these results to more general populations, or other ethnic groups for which fasting glucose screening test validity can vary. Large epidemiological and clinical studies are necessary to examine the relationships between fasting plasma glucose and insulin concentrations in other ethnicities, particularly high-risk populations. As is often the case for population-based research, the diagnostic or clinical practice implications of our observations are limited as it cannot be interpreted that a similar progression in insulin values will occur as fasting plasma glucose increases in individuals.

\section{Conclusion}

In this population-based sample of Aboriginal Australians, second-phase insulin response peaked at a fasting plasma glucose value of $7.0 \mathrm{mmol} / \mathrm{l}$. These epidemiological data and statistical analyses illustrate the relationships between fasting and post-challenge insulin concentrations and fasting plasma glucose levels and support the expanding basic and clinical knowledge base regarding physiological mechanisms underlying the development of diabetes type 2 . The results support current recommendations for diabetes screening in Aboriginal populations that identify fasting glucose of $7.0 \mathrm{mmol} / \mathrm{l}$ and above as diagnostic of diabetes $[16,37]$. The statistical procedures used, and the large sample, improve on the results of earlier research. Additional research is needed to improve understanding of the dynamics of insulin secretion and insulin action at various normal, pre-diabetic and diabetic states, and to understand variations in these processes across different high- and low-risk ethnic groups.

\section{Competing Interests}

The authors declare that they have no competing interests.

\section{Authors' contributions}

$\mathrm{MD}$ and KOD conceived the study. KOD, RM and KR collected the 
data. GZ and MD conducted the statistical analyses. MD and SJK interpreted the results. MD wrote the article. KOD, RM, KR, GZ and SJK provided important critical commentary. All authors approved the final version of the article

\section{Acknowledgements}

The authors thank all of the community councils, community participants and health services personnel involved in this research. We also acknowledge the many others who contributed in fieldwork and technical assistance. We thank Elisabeth Marks, MPH for assistance in reviewing and contributing literature to prior versions of the manuscript.

\section{Publication history}

Received: 11-June-2012 Accepted: 27-July-2012

Published: 11-Aug-2012

\section{References}

1. The Expert Committee on the Diagnosis and Classification of Diabetes Mellitus, Report of the expert committee on the diagnosis and classification of diabetes mellitus. Diabetes Care, 2002. 25 Suppl 1: p. S5-20.

2. World Health Organization, Definition, Diagnosis, and Classification of Diabetes Mellitus and Its Complications: Report of a WHO Consultation. Part 1: Diagnosis and Classification of Diabetes Mellitus. 1999, World Health Organization: Geneva. I Link

3. Daniel M, Rowley KG, McDermott R, O'Dea K: Diabetes and impaired glucose tolerance in Aboriginal Australians: prevalence and risk. Diabetes Res Clin Pract 2002; 57;(1.);23-33. | Article | PubMed

4. Yu CH, Zinman B: Type $\mathbf{2}$ diabetes and impaired glucose tolerance in aboriginal populations: a global perspective. Diabetes Res Clin Pract 2007; 78;(2.);159-70. | Article | PubMed

5. Daniel M, Rowley KG, McDermott R, Mylvaganam A, O'Dea K: Diabetes incidence in an Australian aboriginal population. An 8-year follow-up study. Diabetes Care 1999; 22;(12.);1993-8. I Article I PubMed

6. Wang Z, Hoy WE, Si D: Incidence of type $\mathbf{2}$ diabetes in Aboriginal Australians: an 11-year prospective cohort study. BMC Public Health 2010; 10;(487. | Article | PubMed Abstract | PubMed Full Text

7. Sato $Y$, Komatsu $M$, Katakura $M$, Ohfusa $H$, Yamada $S$, Yamauchi $K$, et al.: Diminution of early insulin response to glucose in subjects with normal but minimally elevated fasting plasma glucose. Evidence for early betacell dysfunction. Diabet Med 2002; 19;(7.);566-71. I Article | PubMed

8. Tanaka Y, Atsumi Y, Asahina T, Hosokawa K, Matsuoka K, Kinoshita J, et al.: Usefulness of revised fasting plasma glucose criterion and characteristics of the insulin response to an oral glucose load in newly diagnosed Japanese diabetic subjects. Diabetes Care 1998; 21;(7.);1133-7. | Article | PubMed

9. Defronzo RA: Pathogenesis of type $\mathbf{2}$ (non-insulin dependent) diabetes mellitus: a balanced overview. Diabetologia 1992; 35;(4.);389-97. | Article I PubMed

10. Savage PJ, Dippe SE, Bennett PH, Gorden P, Roth J, Rushforth NB, et al.: Hyperinsulinemia and hypoinsulinemia. Insulin responses to oral carbohydrate over a wide spectrum of glucose tolerance. Diabetes 1975; 24;(4.);362-8. I PubMed

11. Zimmet $P$, Whitehouse $S$, Alford F, Chisholm D: The relationship of insulin response to a glucose stimulus over a wide range of glucose tolerance. Diabetologia 1978; 15;(1.);23-7. | Article | PubMed
12. DeFronzo RA, Bonadonna RC, Ferrannini E: Pathogenesis of NIDDM. A balanced overview. Diabetes Care 1992; 15;(3.);318-68. | Article | PubMed

13. Godsland IF, Jeffs JA, Johnston DG: Loss of beta cell function as fasting glucose increases in the non-diabetic range. Diabetologia 2004; 47;(7.);1157-66. | Article | PubMed

14. Utzschneider KM, Prigeon RL, Carr DB, Hull RL, Tong J, Shofer JB, et al.: Impact of differences in fasting glucose and glucose tolerance on the hyperbolic relationship between insulin sensitivity and insulin responses. Diabetes Care 2006; 29;(2.);356-62. | Article | PubMed

15. Dowse GK, Zimmet PZ, Alberti KG, Brigham L, Carlin JB, Tuomilehto J, et al.: Serum insulin distributions and reproducibility of the relationship between 2-hour insulin and plasma glucose levels in Asian Indian, Creole, and Chinese Mauritians. Mauritius NCD Study Group. Metabolism 1993; 42;(10.);1232-41. | Article I PubMed

16. Daniel M, Rowley KG, O'Dea K: Fasting criteria for screening: test properties and agreement with glucose tolerance. Diabetes Res Clin Pract 2002; 58;(2.);139-48. | Article | PubMed

17. Rowley KG, Daniel M, Skinner K, Skinner M, White GA, O'Dea K: Effectiveness of a community-directed 'healthy lifestyle' program in a remote Australian aboriginal community. Aust N Z J Public Health 2000; 24;(2.);136-44. | Article | PubMed

18. McDermott R, Rowley KG, Lee AJ, Knight S, O'Dea K: Increase in prevalence of obesity and diabetes and decrease in plasma cholesterol in a central Australian aboriginal community. Med J Aust 2000; 172;(10.);480-4. | Article | PubMed

19. Rowley KG, Gault A, McDermott R, Knight S, McLeay T, O’Dea K: Reduced prevalence of impaired glucose tolerance and no change in prevalence of diabetes despite increasing $\mathrm{BMI}$ among Aboriginal people from a group of remote homeland communities. Diabetes Care 2000; 23;(7.);898-904. | Article | PubMed

20. O'Dea K, Lion RJ, Lee A, Traianedes K, Hopper JL, Rae C: Diabetes, hyperinsulinemia, and hyperlipidemia in small aboriginal community in northern Australia. Diabetes Care 1990; 13;(8.);830-5. | Article | PubMed

21. O'Dea K, White NG, Sinclair AJ: An investigation of nutrition-related risk factors in an isolated Aboriginal community in northern Australia: advantages of a traditionally-orientated life-style. Med J Aust 1988; 148;(4.);177-80. I PubMed

22. McLennan, W. and R. Madden, The Health and Welfare of Australia's Aboriginal and Torres Strait Islander Peoples. 1997, Canberra, ACT: Australian Bureau of Statistics, Australian Government Publishing Service.

23. Robbins DC, Andersen L, Bowsher R, Chance R, Dinesen B, Frank B, et al.: Report of the American Diabetes Association's Task Force on standardization of the insulin assay. Diabetes $1996 ; 45 ;(2.) ; 242-56$. | Article | PubMed

24. Australian Bureau of Statistics, National Health Survey: Aboriginal and Torres Strait Islander Results. 2001, Australian Bureau of Statistics: Canberra.

25. Mathur, S., I. Gajanayake, and G. Hodgson, Diabetes as a Cause of Death, Australia, 1997 and 1998. . 2000, Australia Institute of Health and Welfare: Canberra.

26. Ozaki K, Okubo M, Mori H, Mito K, Hara H, Kohno N: Decreased insulin secretion and dyslipidemia coexist in subjects with impaired fast- 
Daniel et al. Journal of Diabetes Research and Clinical Metabolism 2012, http://www.hoajonline.com/journals/pdf/2050-0866-1-8.pdf

ing glucose. Diabetes Res Clin Pract 2002; 55;(2.);159-64. | Article |

PubMed

27. Gastaldelli A, Ferrannini E, Miyazaki Y, Matsuda M, DeFronzo RA: Betacell dysfunction and glucose intolerance: results from the San Antonio metabolism (SAM) study. Diabetologia 2004; 47;(1.);31-9. | Article | PubMed

28. Pratley RE, Weyer C: The role of impaired early insulin secretion in the pathogenesis of Type II diabetes mellitus. Diabetologia 2001; 44;(8.);929-45. | Article I PubMed

29. Report of the Expert Committee on the Diagnosis and Classification of Diabetes Mellitus. Diabetes Care 1997; 20;(7.);1183-97. | PubMed

30. Abdul-Ghani MA, Jenkinson CP, Richardson DK, Tripathy D, DeFronzo RA: Insulin secretion and action in subjects with impaired fasting glucose and impaired glucose tolerance: results from the Veterans Administration Genetic Epidemiology Study. Diabetes 2006; 55;(5.);1430-5. | Article I PubMed

31. Fukushima M, Usami M, Ikeda M, Nakai Y, Taniguchi A, Matsuura T, et al.: Insulin secretion and insulin sensitivity at different stages of glucose tolerance: a cross-sectional study of Japanese type 2 diabetes. Metabolism 2004; 53; (7.);831-5. | Article | PubMed

32. Nishi Y, Fukushima M, Suzuki H, Mitsui R, Ueda N, Taniguchi A, et al.: Insulin secretion and insulin sensitivity in Japanese subjects with impaired fasting glucose and isolated fasting hyperglycemia. Diabetes Res Clin Pract 2005; 70;(1.);46-52. | Article I PubMed

33. Suzuki H, Fukushima M, Usami M, Ikeda M, Taniguchi A, Nakai Y, et al.: Factors responsible for development from normal glucose tolerance to isolated postchallenge hyperglycemia. Diabetes Care 2003; 26;(4.);1211-5. | Article | PubMed

34. Weyer C, Bogardus C, Pratley RE: Metabolic characteristics of individuals with impaired fasting glucose and/or impaired glucose tolerance. Diabetes 1999; 48;(11.);2197-203. | Article | PubMed

35. Reaven GM, Hollenbeck CB, Chen YD: Relationship between glucose tolerance, insulin secretion, and insulin action in non-obese individuals with varying degrees of glucose tolerance. Diabetologia 1989; 32;(1.);52-5. | Article I PubMed

36. Amoah AG, Schuster DP, Gaillard T, Osei K: Insulin resistance, beta cell function and cardiovascular risk factors in Ghanaians with varying degrees of glucose tolerance. Ethn Dis 2002; 12;(4.);S3-10-7. I Article | PubMed

37. Central Australian Rural Practitioners Association (CARPA), CARPA Standard Treatment Manual, 5th Edition. 2009, Alice Springs: Central Australian Rural Practitioners Association. 